\title{
Wann soll die orale Antikoagulation eingeleitet werden?
}

Fragestellung: Wann ist der beste Zeitpunkt, um bei einem Patienten, der bei Vorhofflimmern einen ischämischen Insult erlitten hat, eine orale Antikoagulation einzuleiten?

Hintergrund: Patienten mit Vorhofflimmern haben ein hohes Schlaganfallrisiko. Dieses kann durch eine orale Antikoagulation reduziert werden. Ein weitgehend ungelöstes Problem ist die Frage, wann es sicher und wirksam ist, nach einem erlittenen ischämischen Insult eine orale Antikoagulation zu beginnen. Durch frühere randomisierte Studien war gezeigt worden, dass eine frühe PTT-wirksame Heparinisierung zwar das Risiko für Rezidivinsulte reduziert, aber gleichzeitig das Blutungsrisiko signifikant erhöht. Die RAF-Studie sollte die Frage beantworten, wie hoch das Risiko von erneuten ischämischen Insulten und schwerwiegenden Blutungskomplikationen nach Einleitung einer oralen Antikoagulation ist, sowie Risikofaktoren für erneute Schlaganfälle und Blutungen identifizieren.

Patienten und Methodik: Die RAF-Studie ist eine prospektive Studie, die zwischen Januar 2012 und März 2014 in 29 Stroke Units in Europa und Asien durchgeführt wurde. Alle Patienten wurden auf einer Stroke Unit behandelt. Bei allen Patienten wurde neben der initialen zerebralen Bildgebung nach 24 bis 72 Stunden eine erneute Bildgebung durchgeführt, um eine

Paciaroni M, Agnelli G, Falocci $\mathrm{N}$ et al. Early recurrence and cerebral bleeding in patients with acute ischemic stroke and atrial fibrillation: Effect of anticoagulation and its timing: The RAF study. Stroke 2015; 46: $2175-82$ hämorrhagische Transformation oder eine parenchymatöse Hirnblutung darzustellen. Der primäre Endpunkt der Studie war die Kombination von Schlaganfall, transienter ischämischer
Attacke, symptomatischer peripherer Embolie, symptomatischen zerebralen und schwerwiegenden extrakraniellen Blutungen innerhalb von 90 Tagen nach dem Schlaganfall. Außerdem wurden die Antithrombotika wie Thrombozytenfunktionshemmer und Antikoagulantien erfasst.

Ergebnisse: Es wurden insgesamt 1.029 Patienten in die Studie eingeschlossen. Den primären Endpunkt erreichten 12,6\% der Patienten. Die Häufigkeit der einzelnen Endpunkte waren 77 $(7,6 \%)$ ischämische Insulte oder transitorische ischämische Attacken (TIA), 37 (3,6\%) symptomatische zerebrale Blutungen und $14(1,4 \%)$ schwerwiegende extrakranielle Blutungen. Nach 90 Tagen waren $50 \%$ der Patienten verstorben oder hatten einen Wert auf der modifizierten Rankin Skala von $\geq 3$.

Prädiktoren für den primären Endpunkt waren ein hoher CHADS2-Score, ein schwerer Schlaganfall gemessen mit der NIHSS, eine große Läsion bei der Bildgebung und die Art der Antikoagulation. In einer adjustierten Regressionsanalyse zeigte sich, dass ein Beginn der Antikoagulation zwischen vier und 14 Tagen nach dem Schlaganfall zu einer signifikanten Reduktion des primären Endpunkts führt. Das Risiko war erhöht, wenn die Behandlung vor dem Tag 4 oder nach dem Tag 14 begann. $7 \%$ der Patienten, die mit oralen Antikoagulanzien behandelt wurden, erreichten den primären Endpunkt verglichen mit $16,8 \%$ der Patienten, die mit niedermolekularem Heparin behandelt wurden, und $12,3 \%$ der Patienten, die initial mit niedermolekularem Heparin und anschließend mit oraler Antikoagulation behandelt wurden.

Schlussfolgerungen: Bei Patienten mit ischämischen Insult und Vorhofflimmern sollte eine orale Antikoagulation zwischen Tag 4 und 14 eingeleitet werden.

\section{- Kommentar von Hans-Christoph Diener, Essen}

\section{Uns bleiben weiterhin nur unsere (nicht datengestützten) Leitlinien}

Diese große Studie aus Europa und Asien ist erschreckend, da offenbar in vielen Stroke Units immer noch eine Antikoagulation mit niedermolekularem Heparin bei Patienten mit ischämischen Insult und Vorhofflimmern durchgeführt wird, obwohl diese Therapie in mehreren randomisierten Studien nicht wirksam war und zu einer erhöhten Rate an Blutungskomplikationen führte [1]. Die Studie zeigt, dass es wohl sicher und wirksam ist, wenn die orale Antikoagulation zwischen Tag vier und 14 begonnen wird.

Leider haben die Autoren versäumt, Prädiktoren zu erarbeiten, bei welchen Patienten eine frühe und bei welchen eine spätere Antikoagulation erfolgen sollte. Daher sind wir als klinische Neurologen weiterhin auf die nicht datengestützten
Empfehlungen zur Initiierung einer Antikoagulation gemäß den europäischen [2] und deutschen Leitlinien angewiesen. Sie empfehlen, dass bei transitorischen ischämischen Attacken (TIA) am ersten Tag antikoaguliert wird, bei leichten Schlaganfällen und geringem morphologischem Defizit nach drei Tagen, bei mittelschweren Schlaganfällen nach sechs Tagen und bei schweren Schlaganfällen mit großem Defizit nach zwölf bis 14 Tagen.

\footnotetext{
Referenzen:

1. Whiteley WN et al. Lancet Neurol 2013; 12: $539-45$

2. Heidbuchel $\mathrm{H}$ et al. Europace 2015
} 